\title{
Avaliação da completude das variáveis epidemiológicas do Sistema de Informação sobre Mortalidade em mulheres com óbitos por câncer de mama na Região Sudeste - Brasil (1998 a 2007)
}

\author{
Evaluation of the plenitude of epidemiological variables \\ of the Information System on Mortality of women with deaths \\ from breast cancer in the Southeast Region - Brazil (1998 - 2007)
}

Janaina Daumas Felix ${ }^{1}$

Eliana Zandonade ${ }^{2}$

Maria Helena Costa Amorim ${ }^{3}$

Denise Silveira de Castro ${ }^{3}$

${ }^{1}$ Hospital Universitário Cassiano Antônio Moraes. Av. Marechal Campos 1355, Santos Dumont. 29140-091 Vitoria ES.

janadaumas@hotmail.com

${ }^{2}$ Departamento de Estatística, Centro de

Ciências Exatas, Universidade Federal do

Espírito Santo

${ }^{3}$ Departamento de

Enfermagem, Centro de

Ciências da Saúde,

Universidade Federal do

Espírito Santo

\begin{abstract}
The study evaluates the data from the Mortality Information System in Espirito Santo, southeastern Brazil in the period from 1998 to 2007. It is a descriptive analytic study based on secondary data. The variables of sex, age, racel color, level of education and marital status in women who died of breast cancer were evaluated. The scores used were excellent (variable shows less than 5\% of incomplete coverage), good (5\% to $10 \%$ ), fair (10\% to $20 \%$ ), poor (20\% to $50 \%$ ) and very poor (50\% or higher). The linear trend equations were calculated using SPSS, version 15.0 for non-plenitude over time. It is essential to include in the education of health professionals, especially in undergraduate and postgraduate studies in medicine, content that develops skills and competencies for systematic registration of epidemiological data, so they can be included in the health information systems.
\end{abstract}

Key words Breast cancer; Information system, Quality
Resumo O estudo avalia a completude dos dados do Sistema de Informação sobre Mortalidade no Espirito Santo, Região Sudeste e Brasil no período de 1998 a 2007. Trata-se de um estudo descritivo analítico baseado em dados secundários. Avaliouse as variáveis de sexo, idade, raça/cor, escolaridade e estado civil em mulheres com óbito por câncer de mama. Utilizou-se os escores excelente (variável apresenta menos de $5 \%$ de preenchimento incompleto), bom (5\% a 10\%), regular (10\% a 20\%), ruim (20\% a $50 \%$ ) e muito ruim (50\% ou mais). Calculou-se as equações de tendência linear para a não completude ao longo do tempo. Os resultados para as variáveis sexo e idade foram excelentes para o Espirito Santo, o Sudeste e o Brasil. Enquanto no Sudeste e no Brasil as variáveis raça/cor e escolaridade têm tendência decrescente para a não completude, no Espírito Santo se mantém estável, mas inadequadas. Para a variável estado civil, a não completude tem tendência crescente no Espírito Santo, e inadequada. Conclui-se que, as declarações de óbito com câncer de mama neste estado apresentam inconsistências, e sugere-se que na formação dos profissionais de saúde, em especial nos cursos de medicina, sejam desenvolvidas habilidades e competências no registro de dados epidemiológicos, para poderem fundamentar os sistemas de informação em saúde.

Palavras-chave Câncer de mama, Sistema de informação, Qualidade 


\section{Introdução}

No Brasil, havia uma diversidade na forma do registro de dados referentes à saúde no país, quando em 1975, o Ministério da Saúde reconhece a importância deste registro e cria o Sistema de Informação em Saúde (SIS), com dados de nascidos vivos, internações hospitalares no Sistema Único de Saúde e mortalidade. Neste novo processo de trabalho, promoveu-se a implantação de um sistema nacional de vigilância epidemiológica e se propôs um modelo único de declaração de óbito (DO), que ofereceu subsídios para a implantação do Sistema de Informação em Mortalidade (SIM), que é o foco central deste artigo.

O SIM foi implantado em 1975 para suprir as falhas do Registro Civil e permitir conhecer o perfil epidemiológico da mortalidade em todo o país ${ }^{1}$.

As estatísticas de mortalidade constituem-se uma importante ferramenta para o conhecimento do perfil epidemiológico de uma população, elaboração de indicadores de saúde, análise de tendências, indicação de prioridades e, consequente, planejamento de ações desse setor ${ }^{2,3}$.

Nos últimos anos, tem se intensificado o interesse pelas análises da mortalidade, dada a importância desta estatística, avaliando os mais diferentes aspectos, tais como cobertura do sistema nas Unidades da Federação e qualidade dos dados $^{1,3,4}$. Apesar de ainda se ter problemas com as informações referentes ao SIM, tais como a existência de sub-registro dos eventos vitais, erros no preenchimento da DO, falhas de cobertura do Sistema e perdas na transmissão dos dados do SIM/DATASUS, elas ainda continuam sendo úteis como fonte de pesquisas.

As informações obtidas a partir das notificações são de grande importância, pois possibilitam a monitorização espaço-temporal de epidemias no país, bem como o acompanhamento da disseminação de doença por categoria de exposição, subsidiando as ações para sua prevenção e controle. Para que a vigilância seja eficiente, é necessário, portanto, que essas informações sejam de boa qualidade 5 .

A qualidade das informações disponibilizadas no SIM depende de fatores internos e externos. Os internos seriam a qualidade do programa do computador para sustentar o software e a capacitação dos profissionais de saúde envolvidos no processo de trabalho, em especial os codificadores de causa base de óbito. Os fatores externos remetem à assistência à saúde da população, se a mesma é adequada ou inexistente, conferindo informações fidedignas sobre a causa da morte ${ }^{5}$. É necessário, portanto, a avaliação do atributo qualidade dos dados, que pode ser mensurado pela avaliação da validade dos dados e da completude dos campos.

O câncer de mama é a segunda maior causa de óbito nos países desenvolvidos, ficando atrás somente das doenças cardiovasculares. Dentre as neoplasias, o câncer de mama é a principal causa de morte nas mulheres ${ }^{6-8}$.

Em virtude do seu caráter contínuo e amplitude nacional, a DO contêm registros de informações com variáveis socioeconômicas e demográficas, que constituem fontes de informação privilegiadas para estudo e monitoramento da desigualdade em saúde.

Nesse sentido, este trabalho tem por objetivo analisar a qualidade do Sistema de Informação em Mortalidade (SIM) no aspecto da completude sobre os óbitos por câncer de mama em mulheres no estado do ES, na Região Sudeste e no Brasil, no período de 1998 a 2007, referentes às variáveis epidemiológicas (idade, sexo, raça/cor, escolaridade e estado civil).

\section{Métodos}

Realizou-se um estudo descritivo analítico baseado em dados secundários. Foram incluídos os óbitos, no período de 1998 a 2007, ocorridos no Estado do Espírito Santo, na Região Sudeste e no Brasil, registrados no (SIM), em mulheres que possuíam como causa base de óbito o câncer de mama, os quais foram obtidos no endereço eletrônico do Departamento de Informática do Sistema Único de Saúde (DATASUS) do Ministério da Saúde9. Dados sobre estimativas populacionais foram obtidos no Instituto Brasileiro de Geografia $(\mathrm{IBGE})^{10}$. Optou-se em estudar os últimos 10 anos do SIM (1998 a 2007), por se tratar de um período recente, e assim, avaliar a qualidade da informação das variáveis em questão na última década.

O período selecionado (1998 a 2007) para cálculo do número de óbitos por câncer de mama em mulheres refere-se ao período de vigência da Décima Revisão da Classificação Internacional de Doenças (CID-10) a qual arrola as categorias C50.0 à C50.9, referentes aos óbitos por neoplasia maligna de mama ${ }^{11}$.

O termo completude refere-se ao grau de preenchimento de campo analisado, mensurado pela proporção de notificações com campo preenchido com categoria distinta daquelas indicadoras de ausência do dado. Campo preenchido no ban- 
co de dados com categoria "ignorada", numeral zero, data ignorada ou termo que indica ausência do dado será considerado incompleto ${ }^{12}$.

Foi realizada uma análise inferencial com regressão linear simples das variáveis demográficas disponíveis no sistema do DATASUS (idade, sexo, estado civil, escolaridade, raça/cor), por meio do conceito de completude dos campos propostos por Romero e Cunha ${ }^{13}$, adaptados pela Comissão Econômica para a América Latina e Caribe (CEPAL), com intuito de analisar a tendência da não completude das variáveis citadas acima.

O escore utilizado possui os seguintes graus de avaliação: excelente, quando a variável apresenta menos de $5 \%$ de preenchimento incompleto; bom (5\% a $10 \%)$; regular $(10 \%$ a $20 \%)$; ruim (20\% a $50 \%)$; muito ruim (50\% ou mais) $)^{13-15}$. Foi analisado o número absoluto e o percentual de não completude das informações das DO, que são a base de informação do SIM, nas localidades selecionadas (ES, Região Sudeste e Brasil).

Para análise dos dados foi utilizado o Programa Excel 7.0. Quanto à apresentação dos resultados, esta ocorreu por meios de tabelas e gráficos, visando sua melhor compreensão. As equações de tendência linear e as estatísticas de ajuste de modelo (valor de R2 e o p-valor do teste F de adequação do modelo) foram obtidas do programa SPSS, versão 15.0. O nível de significância adotado foi de $5 \%$.

No que se refere aos aspectos éticos, destacase que todo o processo de pesquisa foi conduzido conforme a Resolução No. 196/96 do Conselho Nacional de Saúde (CNS), tendo sido o pro- jeto aprovado pelo Comitê de Ética e Pesquisa da Universidade Federal do Espírito Santo.

\section{Resultados}

A análise do SIM, no período compreendido entre 1998 a 2007, no estado do Espírito Santo (ES), permitiu identificar a ocorrência de 1.455 óbitos em mulheres por câncer de mama. A incidência neste período variou de 17,37 (ano 1998) a 38,48 (ano 2007) casos por 100.000 habitantes.

Na Região Sudeste houve 53.009 óbitos em mulheres pela mesma causa base de morte, sendo que a taxa de mortalidade neste período variou de 27,55 (ano 1998) a 35,32 (ano 2007) por 100.000 habitantes. Para o Brasil, foram 93.279 óbitos por esta causa. A taxa de mortalidade por câncer de mama em mulheres neste período variou de 9,26 (ano 1998) a 12,82 (ano 2007) por 100.000 habitantes.

No período de estudo foi observado total completude dos dados para as variáveis de sexo e idade, nessa população (ES, Sudeste e Brasil).

A Tabela 1 apresenta a frequência absoluta e percentual dos dados ignorados do SIM para câncer de mama em mulheres das variáveis "raça/ cor", "escolaridade" e "estado civil", no Espírito Santo, de 1998 a 2007.

Para a variável "raça/cor", a variabilidade de dados ignorados de informações das DO por câncer de mama em mulheres no Espírito Santo se mantém entre $18 \%$ a $35 \%$, o que confere um padrão de qualidade ruim. A variável "escolaridade"

Tabela 1. Frequência absoluta e percentual dos dados ignorados do SIM para câncer de mama em mulheres, para as variáveis raça/cor, escolaridade e estado civil, ES, 1998 a 2007.

\begin{tabular}{|c|c|c|c|c|c|c|c|}
\hline \multirow{2}{*}{ Ano } & \multicolumn{2}{|c|}{ Cor/raça } & \multicolumn{2}{|c|}{ Escolaridade } & \multicolumn{2}{|c|}{ Estado civil } & \multirow{2}{*}{ Total de Caso } \\
\hline & $\%$ & Escore & $\%$ & Escore & $\%$ & Escore & \\
\hline 1998 & $35 \%$ & RU & $79 \%$ & MR & $3 \%$ & $\mathrm{E}$ & 84 \\
\hline 1999 & $27 \%$ & RU & $48 \%$ & RU & $3 \%$ & $\mathrm{E}$ & 96 \\
\hline 2000 & $18 \%$ & $\mathrm{R}$ & $42 \%$ & $\mathrm{RU}$ & $5 \%$ & B & 107 \\
\hline 2001 & $30 \%$ & RU & $47 \%$ & RU & $7 \%$ & B & 128 \\
\hline 2002 & $25 \%$ & RU & $62 \%$ & MR & $10 \%$ & B & 155 \\
\hline 2003 & $34 \%$ & RU & $51 \%$ & MR & $11 \%$ & $\mathrm{R}$ & 172 \\
\hline 2004 & $21 \%$ & RU & $49 \%$ & RU & $10 \%$ & B & 183 \\
\hline 2005 & $28 \%$ & RU & $55 \%$ & MR & $10 \%$ & $\mathrm{~B}$ & 177 \\
\hline 2006 & $26 \%$ & RU & $58 \%$ & MR & $9 \%$ & B & 178 \\
\hline 2007 & $30 \%$ & RU & $59 \%$ & MR & $11 \%$ & $\mathrm{R}$ & 175 \\
\hline
\end{tabular}

$\mathrm{E}=$ excelente, $\mathrm{B}=$ bom, $\mathrm{R}=$ regular, $\mathrm{RU}=$ ruim, $\mathrm{MR}=$ muito ruim 
chega a ter parâmetros entre $42 \%$ a $79 \%$ de não completude, se colocando no patamar de qualidade muito ruim da informação. Em relação à variável "estado civil" no ES, a mesma se apresenta com qualidade excelente nos anos de 1998 e 1999 caindo para regular com o passar do tempo.

A Tabela 2 apresenta os resultados da análise de tendências dos dados ignorados dos óbitos por câncer de mama para as variáveis "raça/cor", "escolaridade" e "estado civil", para o estado do Espírito Santo, Sudeste e Brasil. De forma geral, no período 1998 a 2007, observa-se uma tendência decrescente de não completude para todas as variáveis no presente estudo nas regiões Sudeste e Brasil. Entretanto, o ES se mantém estável na tendência a não completude dos dados na variá- vel "raça/cor" e escolaridade e com tendência crescente na variável "estado civil" $(\mathrm{p}<0,001)$.

Nos dados do SIM, no período de 1998 a 2007, a análise da variável "raça/cor" por óbitos em mulheres por câncer de mama, demonstrou claramente a redução de dados ignorados na região Sudeste ( $\mathrm{p}=0,037$ com decrescimento de $58 \%$ ) e no Brasil ( $\mathrm{p}=0,005$ com decrescimento de 75\%). Entretanto, o ES, mesmo pertencendo à Região Sudeste, não acompanha esse declínio na tendência em dados ignorados, podendo ser visualizado na Figura 1.

Em relação à variável "escolaridade”, o ES segue a mesma linha de tendência da variável anterior, permanecendo estável e se contrapondo em relação ao Sudeste $(\mathrm{p}=0,029$ com decrescimento

Tabela 2. Resultado da análise de tendências dos dados ignorados dos óbitos por câncer de mama em mulheres por raça/cor, escolaridade, estado civil e local de ocorrência, período 1998 a 2007.

\begin{tabular}{|c|c|c|c|c|c|c|}
\hline Variável & Local & Modelo & $\mathbf{R}^{2}$ & p-valor & Tendência & Crescimento \% \\
\hline \multirow[t]{3}{*}{ Raça/cor } & ES & $y=0,281+0,001 x$ & 0,005 & 0,849 & estável & - \\
\hline & Sudeste & $y=0,130-0,010 x$ & 0,491 & 0,024 & decrescente & $-58,0$ \\
\hline & Brasil & $y=0,189-0,017 x$ & 0,640 & 0,005 & decrescente & $-75,0$ \\
\hline \multirow[t]{3}{*}{ Escolaridade } & ES & $y=0,567-0,003 x$ & 0,008 & 0,810 & estável & - \\
\hline & Sudeste & $y=0,523-0,030 x$ & 0,439 & 0,037 & decrescente & $-62,0$ \\
\hline & Brasil & $y=0,547-0,030 x$ & 0,474 & 0,028 & decrescente & $-62,0$ \\
\hline \multirow[t]{3}{*}{ Estado Civil } & ES & $y=0,029+0,009 x$ & 0,740 & 0,001 & crescente & $+75,5$ \\
\hline & Sudeste & $y=0,013+0,001 x$ & 0,175 & 0,230 & estável & - \\
\hline & Brasil & $y=0,033+0,001 x$ & 0,165 & 0,244 & estável & - \\
\hline
\end{tabular}

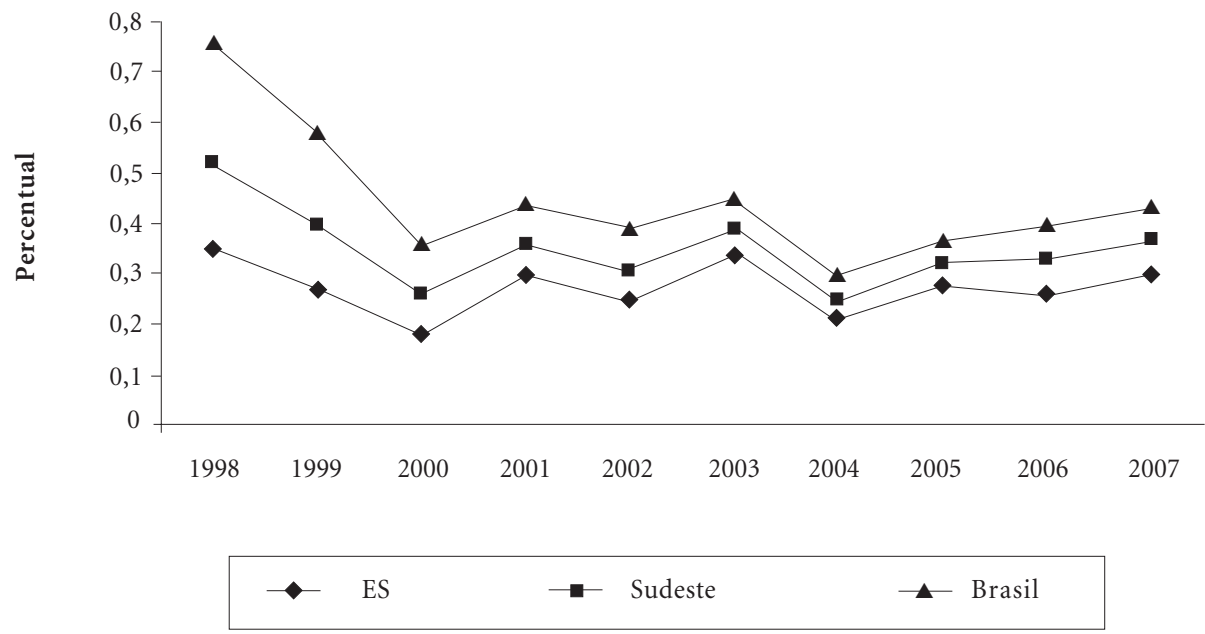

Figura 1. Percentual de não completude da variável raça cor para dados de Mortalidade de Câncer de mama, de 1998 a 2007. 
de $62 \%$ ), e ao Brasil ( $\mathrm{p}=0,018$ com decrescimento de $62 \%$ ), podendo ser visualizado na Figura 2.

Em relação à variável "estado civil", o ES segue com tendência crescente ( $\mathrm{p}=0,001$ com crescimento de $75 \%$ ), enquanto que a região Sudeste e o Brasil decrescem na tendência. Pode ser visualizado na Figura 3.

\section{Discussão}

No Brasil, as estimativas para o ano de 2010 apontavam que ocorreriam 466.730 casos novos de câncer. Sendo que no caso do câncer de mama o esperado no país era de 49.240, com um risco estimado de 49 casos cada 100 mil mulheres. No

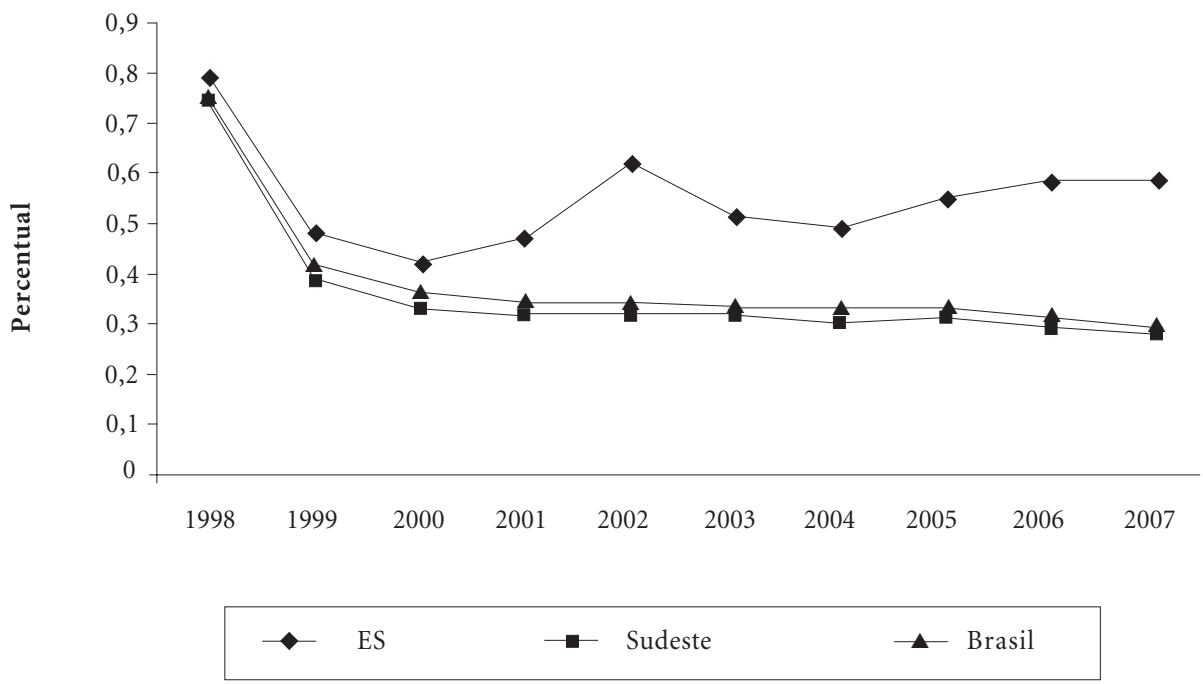

Figura 2. Percentual de não completude da variável escolaridade para dados de Mortalidade de Câncer de mama, de 1998 a 2007.

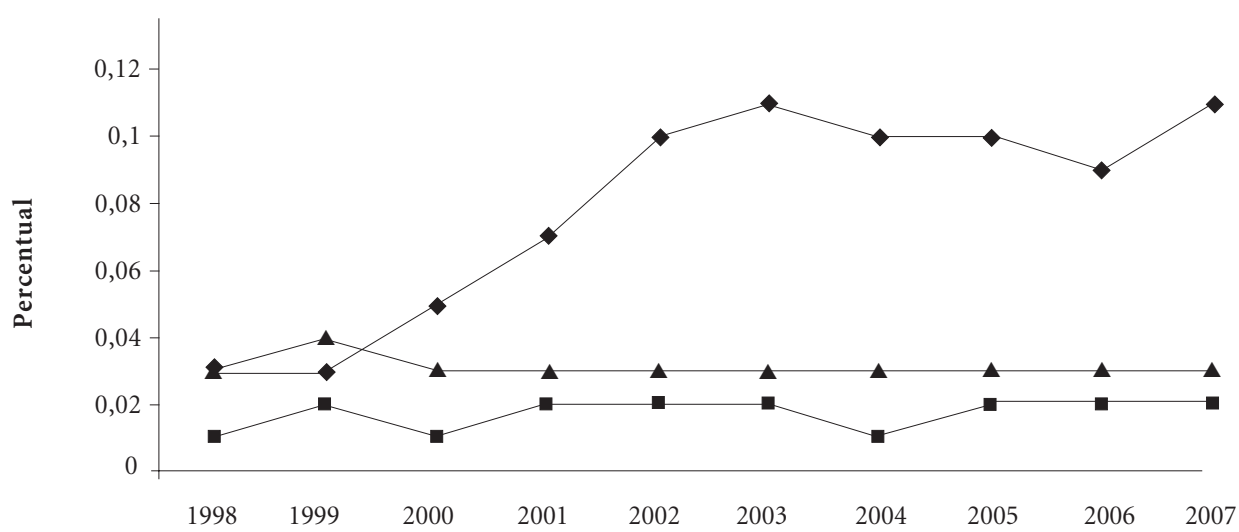

Figura 3. Percentual de não completude da variável estado civil para dados de Mortalidade de Câncer de mama, de 1998 a 2007 
Sudeste, esse tipo de câncer é o mais frequente nas mulheres, com uma taxa bruta incidente de 64,54 casos para 100 mil habitantes. No ES, desconsiderando os casos de tumores de pele nãomelanoma, esse tipo de câncer é o mais frequente nas mulheres, com uma taxa bruta de $44,14 \mathrm{ca}-$ sos para cada 100 mil habitantes ${ }^{16}$.

Devido a essas estimativas, o câncer de mama é considerado um grave problema de saúde pública, não só pelo número crescente de casos diagnosticados a cada ano, mas também pelo investimento financeiro que é solicitado para solucionar as questões de diagnóstico e tratamento. Por isso se faz necessário o conhecimento do perfil epidemiológico dessa população para assim propor medidas de controle a grupos vulneráveis.

No país, a disponibilidade e a qualidade das estatísticas de óbitos são fatores que limitam os estudos sobre mortalidade ${ }^{17}$.

Há pelo menos 30 anos, sistemas de informação vêm sendo criados pelo Ministério da Saúde (MS), com o objetivo de mostrar a evolução das estatísticas vitais. Entretanto, ainda se pode ouvir expressões como "não há dados disponíveis" ou "os dados estão incompletos" ${ }^{1}$, colocando em cheque a confiabilidade dos dados dos sistemas de informação do nosso país. A proporção de "causas mal definidas" tem sido utilizadas para avaliar a qualidade dos dados de mortalidade por causas na DO, quanto maior a proporção de óbitos por causas mal definidas em uma distribuição, menor a exatidão dessas estatísticas ${ }^{2}$.

Embora tenha ocorrido uma melhora da cobertura pelo SIM e por outros sistemas de informação em todo território brasileiro, ainda existe elevado número de informações ignoradas ou não preenchidas.

A ausência de informação nas variáveis sexo e idade nas estatísticas vitais do país vem se mantendo sempre em níveis baixos de não completude (menos de $1 \%$ ), o que corrobora com os resultados apresentados neste estudo, onde não foram encontrados não completude para essas variáveis na população aqui estudada, permitindo, assim, que essas possam ser analisadas do ponto de vista epidemiológico ${ }^{1}$.

A variável "raça/cor" foi introduzida no SIM em 1995, mas somente a partir do ano de 2000 o MS passou a trabalhar este dado com o intuito de melhorar as estatísticas vitais, podendo investigar dessa forma diferenças entre brancos, negros e pardos ${ }^{18}$, e nos últimos anos tem se intensificado o interesse pelas análises acerca de raça/ cor e etnia como fatores determinantes de desi- gualdades na saúde no Brasil ${ }^{19}$. A análise e a interpretação desses dados ainda são escassas na literatura epidemiológica nacional, ao contrário de outros países, como os Estados Unidos e Inglaterra ${ }^{20}$.

Porém a coleta desses dados ainda se mostra deficiente. São bastante claras as limitações para a classificação da raça/cor em recém-nascidos e em óbitos, pois o procedimento mais adequado e amplamente recomendado é o da autoclassificação.

Cardoso et al. ${ }^{21}$ em 2005, comprovaram em um trabalho de análise do SIM e do SINASC na mortalidade infantil segundo raça/cor em todo o Brasil, que houve uma progressiva redução nas proporções de óbitos com raça/cor não informada, porém essa melhoria ainda não se dá de maneira homogênea nas Unidades da Federação, os autores puderam demonstrar que o ES, mesmo sendo um estado pertencente à região Sudeste, possui um dos piores índices do país na não completude de dados referente à raça/cor. $\mathrm{O}$ que evidencia desigualdades intrarregionais nas declarações de óbitos.

No documento "A construção da Política de Informação e Informática em Saúde do Sistema Único de Saúde", de 2003, está muito claro que as características sociodemográficas, tais como idade, gênero, raça/etnia, escolaridade, ocupação e classe social poderão ser utilizadas para a realização de estudos que objetivem a redução das desigualdades em saúde e a ampliação do acesso da população a serviços de qualidade, oportunos e humanizados ${ }^{22}$.

$\mathrm{Na}$ variável grau de escolaridade, a não completude decresce no Sudeste e no Brasil, enquanto que no ES permanece estável sem tendência de crescimento. Poucos são os estudos que se baseiam nessa variável, pois a sua não completude é muita elevada em todo território brasileiro. Santa Helena e Rosa ${ }^{23}$ verificaram a existência de mau preenchimento das variáveis ocupação e escolaridade materna, pontuando que de modo geral essas variáveis são preenchidas pelo pessoal da área administrativa.

Jobim e Aerts, em um trabalho de análise da mortalidade infantil e fatores associados, demonstraram uma associação entre mortalidade infantil e baixa escolaridade materna, podendo dessa maneira identificar grupos de vulnerabilidade a esse óbito ${ }^{24}$.

Nesse mesmo contexto, Schneider e D'Orsi, em estudo recente em uma coorte histórica de mulheres com câncer de mama diagnosticadas no período de $1^{\circ}$ de janeiro de 2000 a 31 de de- 
zembro de 2002, detectaram que a sobrevida daquelas com grau de escolaridade superior com esta neoplasia era maior do que as que apresentavam a mesma doença, porém com nível de escolaridade inferior ${ }^{6}$.

Molina e Luca também puderam observar em um estudo de análise das oportunidades de diagnóstico precoce para câncer de mama, que as mulheres com menos de quatro anos de estudos sabiam menos sobre a periodicidade do autoexame do que mulheres que estudavam nove anos ou mais, encontrando significância estatística ${ }^{25}$.

Quanto à variável "estado civil", o ES mantém uma tendência de crescimento a não completude $(\mathrm{ES}+75 \%$ com $\mathrm{p}<0,001)$. Há de se dizer que a responsabilidade do preenchimento da DO é exclusiva do médico. No que diz respeito a pessoas acometidas por câncer, se pressupõe que se trata de um processo de adoecimento de longo curso de duração, que requer uma interação entre médico e paciente, oferecendo dessa forma subsídios para o preenchimento adequado e completo dos dados epidemiológicos em questão, pois na maioria dos casos o médico que preenche a DO, é o mesmo profissional que acompanha o paciente e que determina suas opções de tratamento.

É ele, profissional médico, portanto depois de formado e principalmente durante a sua formação, que deve ser sensibilizado no sentido de valorizar a real importância do correto preenchimento da DO e educado quanto à maneira adequada de fazê-lo.

Pode-se dizer que houve um imenso empenho do MS, Secretarias Estaduais e Municipais e várias outras entidades do governo e da Saúde, no que diz respeito a melhora na DO no quesito causa base da mortalidade, pois houve uma redução significativa das "causas mal definidas" e por "diagnósticos incompletos”, mas ainda há um longo caminhar a percorrer, pois existem questões epidemiológicas que necessitam ser melhoradas, permitindo que se faça um delineamento do padrão de mortalidade por câncer de mama na população.

A melhoria da cobertura do SIM e a diminuição do número de causas bases mal definidas que vem ocorrendo ao longo do território brasileiro, hoje já é suficiente. É necessário que se obtenha o empenho das entidades envolvidas na melhora da captação dos dados. Como exemplo, tem-se a cidade de São Paulo (SP), ao criar em 1989 o Programa de Aprimoramento das Informações de Mortalidade (PROAIM), com o objetivo de for- necer informações de mortalidade necessárias aos diagnósticos de saúde, vigilância epidemiológica e avaliação dos serviços de saúde, tem acesso diariamente a cerca de 200 DO relativas à óbitos ocorridos na capital paulista no dia anterior. Essa agilidade permite o desencadeamento de ações imediatas, entre outros aspectos, aqueles relacionados à melhoria da qualidade da informação, pois esse programa envia cartas aos médicos declarantes em todos os casos onde não se consegue definir uma real precisão da causa morte, essa metodologia é usada como orientação para o correto preenchimento da $\mathrm{DO}^{26}$.

A Secretaria Municipal de Saúde do RecifePE adotou três linhas de ação, a primeira foi a capacitação de pessoal, depois o incentivo à capacitação sistemática de dados não registrados nas DO e por último a análise da coerência dos bancos de dados. Essas ações englobam desde a coleta diária da DO nos cartórios do município, contato telefônico com os médicos declarantes e com as unidades de saúde, até parceria com a Corregedoria Geral da Justiça e a Secretaria de Justiça, permitindo a sensibilização, a orientação e a capacitação dos Recursos Humanos envolvidos no processamento da DO. Desse modo, pôde ser verificada uma melhoria bastante adequada das informações.

É prioritário o papel dos comitês de mortalidade das instituições de saúde, de modo que além de realizarem suas atividades, como estudar as formas de se evitar o óbito nas Instituições, também possam fornecer estatísticas da não completude das informações contidas na DO para subsidiar ações educativas dos médicos declarantes, visando um melhor preenchimento do formulário de atestado de óbito. E que as Secretarias Estaduais de Saúde, junto aos Comitês de Mortalidade Institucionais, devolvam às instituições as vias da DO pertencente às Secretarias, com dados ignorados e incompletos, com prazos determinados de devolução com preenchimentos adequados pelos médicos declarantes.

A busca pela qualidade da DO existe desde as décadas de 50 e 60, mas parte da classe médica ainda não dá a devida importância ao preenchimento do atestado, tão necessário no contexto da epidemiologia, do planejamento e da programação de saúde ${ }^{27}$.

É preciso fortalecer no currículo das escolas de medicina, desde o internato à residência médica, temas relacionados às estatísticas de mortalidade, seus atributos e a importância do preenchimento adequado de uma DO. 


\section{Conclusão}

Os resultados ressaltam que a declaração de óbito no Estado do Espírito Santo apresenta inadequações, pois possui ausência de informação nas variáveis epidemiológicas. Desse modo, é necessária a construção de registro de dados consistentes e fidedignos, uma vez que esses poderão subsidiar pesquisas e políticas institucionais públicas.

Torna-se imprescindível incluir no processo de formação dos profissionais de saúde, em especial nos cursos de graduação e de pós-graduação em medicina, conteúdos que desenvolvam habilidades e competências para sistematizar o registro de dados epidemiológicos, de modo que possam fundamentar os sistemas de informação em saúde.

Vale destacar ainda a importância do envolvimento dos gestores institucionais, municipais e estaduais nessa questão e sensibilizá-los quanto à relevância da qualidade dos dados sobre mortalidade, na construção de indicadores que demonstrem uma melhoria da cobertura e da qualidade das informações, para assim, possibilitar as discussões sobre desigualdades em saúde no país.

\section{Colaboradores}

JD Felix trabalhou na concepção teórica, na elaboração e na redação final do artigo e E Zandonade, MHC Amorim e DS Castro contribuíram na redação e na revisão final do artigo. 


\section{Referências}

1. Mello Jorge MHP, Laurenti R, Gotlieb SLD. Análise da qualidade das estatísticas vitais brasileiras: a experiência de implantação do SIM e do SINASC. Cien Saude Colet 2007; 12(3):643-654.

2. Laurenti R, Mello Jorge MHP, Gotlieb SLD. A confiabilidade dos dados de mortalidade e morbidade por doenças crônicas não-transmissíveis. Cien Saude Colet 2004; 9(4):909-920.

3. Haraki CAP, Goltilieb SLD, Laurenti R. Confiabilidade do sistema de informações sobre mortalidade em município do Sul do Estado de São Paulo. Rev Bras Epidemiologia 2005; 8(1):19-24.

4. Gomes FBC. Sistema de informações sobre mortalidade: considerações sobre a qualidade dos dados. Infor Epidemio SUS 2002;11(1):5-6.

5. Cerqueira ACB, Sales CMM, Lima R, Silva MZ, Vieira RCA, Brioschi AP, Moreira-Silva SF, Lima LHM, Miranda AE, Maciel ELN. Completude do sistema de informação de agravos de notificação compulsória de gestante HIV positivo entre 2001 e 2006, no Espírito Santo, Brasil. UFES Rev Odont 2008; 10(1):33-37.

6. Schneider IJC, D’Orsi E. Sobrevida em cinco anos e fatores prognósticos em mulheres com câncer de mama em Santa Catarina, Brasil. Cad Saude Publica 2009; 25(6):1285-1296.

7. Wünch Filho V, Moncau JE. Mortalidade por câncer no Brasil 1980-1995: padrões regionais e tendências temporais. Rev Assoc Med 2002; 48(3):250-257.

8. Mathias TAF, Mello Jorge MHP. Evolução da mortalidade por neoplasias em idosos em município do estado do Paraná,1979-1998. Cienc Cuid Saúde 2006; 5(Supl.):57-67.

9. Brasil. Ministério da Saúde [homepage na Internet]. Departamento de Informática do SUS - DATASUS. Informações de Saúde. Estatísticas Vitais de Morbi-Mortalidade. [acessado 2009 dez 15]. Disponível em: http://tabnet.datasus.gov.br/cgi/ tabcgi.exe?sim/cnv/obtes.def

10. Instituto Brasileiro de Estatística e Geografia (IBGE). Censo Demográfico de 2000. Rio de Janeiro: Instituto Brasileiro de Geografia e Estatística (IBGE); 2002.

11. Organização Mundial de Saúde (OMS). Manual da classificação internacional de doenças e problemas relacionados à saúde. $10^{\mathrm{a}}$ revisão. São Paulo: Centro Colaborador da Organização Mundial de Saúde (OMS) para Classificação de Doenças em Português; 1975.

12. Glatt R. Análise da qualidade da base de dados de AIDS do Sistema de Informação de Agravos de Notificação (SINAN) [dissertação]. Rio de Janeiro; Escola Nacional de Saúde Pública; 2005.

13. Romero DE, Cunha AB. Avaliação da qualidade das variáveis sócio-econômicas e demográficas dos óbitos de crianças menores de um ano registrados no Sistema de Informação Sobre Mortalidade do Brasil(1996/2001). Cad Saude Publica 2006; 22(3):673-684.

14. Comisión Econômica para América Latina y el Caribe. Gestión orientada a asegurar la calidad de los dados en los institutos nacionales de Estadística. Santiago de Chile: Comisión Económica para América Latina y el Caribe; 2003.
15. Romero DE, Cunha AB. Avaliação da qualidade das variáveis epidemiológicas e demográficas do Sistema de Informações sobre Nascidos Vivos,2002. Cad Saude Publica 2007; 23(3):701-714.

16. Brasil. Ministério da Saúde (MS). Secretaria de Atenção à Saúde. Instituto Nacional de Câncer. Coordenação de Prevenção e Vigilância de Câncer. Estimativas 2010: Incidência de Câncer no Brasil. Rio de Janeiro: Instituto Nacional de Câncer (INCA); 2009.

17. Vasconcelos AMN. A qualidade das estatísticas de óbito no Brasil. Rev Bras Est Populacionais 1998; 15(1):115-124.

18. Brasil. Fundação Nacional de Saúde da População Negra no Brasil. Contribuições para a promoção da equidade. Brasília: Fundação Nacional de Saúde (FUNASA); 2005.

19. Travassos C, Williams DR. The concept and measurement of race and their relationship to public health; a review focused on Brazil and United States. Cad Saude Publica 2004; 20(3):660-678.

20. Chor D, Lima CRA. Aspectos epidemiológicos das desigualdades raciais em saúde no Brasil. Cad Saude Publica 2005; 21(5):1586-1594.

21. Cardoso AM, Santos VR, Coimbra Junior CEA. Mortalidade infantil segundo raça/cor no Brasil: o que dizem os sistemas nacionais de informação? Cad Saude Publica 2005; 21(5):1602-1608.

22. Brasil. Ministério da Saúde. Secretaria Executiva. Departamento de Informação e Informática do SUS [homepage na Internet]. A construção da política nacional de informação e informática em saúde: proposta versão 2.0: (inclui deliberações da 12. ${ }^{a}$ Conferência Nacional de Saúde). Brasília: Ministério da Saúde; 2004. [acessado 2012 mar 15]. Disponível em: http://bvsms.saude.gov.br/bvs/publicacoes/PoliticaInformacaoSaude29_03_2004.pdf

23. Santa Helena ET, Rosa MB. Avaliação da qualidade das informações relativas aos óbitos em menores de um ano em Blumenau, 1998. Rev Bras Saúde Mater Infant 2003; 3(1):75-83.

24. Jobim R, Aerts D. Mortalidade infantil evitável e fatores associados em Porto Alegre, Rio Grande do Sul, Brasil, 2000-2003. Cad Saude Publica 2008; 24(1):179-187.

25. Molina L, Luca IDL. Análise das oportunidades de diagnóstico precoce para as neoplasias malignas de mama.Rev. Assoc.Med.Brasileira,2003;49(2):185-190.

26. Laurenti R, Mello Jorge MHP, Gotlieb SLD. O sistema de informações sobre mortalidade: passado, pre-

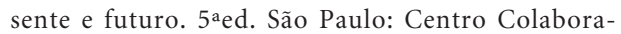
dor da OMS para a Família de Classificações em Português; 2006.

27. Oliveira BZ, Gotlieb SLD, Laurenti R, Mello Jorge MHP. Mortalidade feminina por hipertensão: análise por causas múltiplas. Rev Bras Epidemiologia 2009; 12(4):556-565.

Artigo apresentado em 17/03/2011

Aprovado em 30/08/2011

Versão final apresentada em 16/09/2011 